\title{
Of virtual groups and their way of communicating
}

\author{
A. I. Teleoacă \\ Faculty of Letters, History and Theology, \\ Applied Modern Languages Department, Galatz, Romania
}

\begin{abstract}
Computers, the Internet and their language have obviously become an important part in people's lives from all over the world, and, consequently, have reached all strata of every civilized society whether social, economic, political or educational. It turns out to be evident that the new language has brought new cultural concepts as well because many computer and Internet terms were coined as a result of a specific cultural angle, and that will include certain American concepts, ideas, habits, objects, and gestures.

This paper will take into consideration some of the culturally-embedded concepts in the language of the Internet that has beyond doubt changed our way of thinking about the future. The method used will be a contrastive study on the American and specifically of globalisation.

In the past 17 years, Romania has encountered a constant technological boom that is to be traced to a variety of domains such as industry, economy, education, mass media, politics, etc. A case in point is the personal computer that has become an irreplaceable tool involved in almost all the activity areas among which the education and mass media systems are continuously benefiting by. Consequently, new concepts, like the so far well-known multimedia technology, user-friendly systems, Internet, Web technologies, cyberspace communities, virtual reality, etc. have been brought up into Romanian culture. Therefore, I will try to develop the first part of the paper into a contrastive cultural analysis between some of the American behavioural patterns, beliefs, values and symbols that are to be perceived when thinking of the Internet, what this means, and how it functions, and the specificity of globalisation under the influence of such powerful technology.
\end{abstract}




\section{The concept of e-connection}

To start with, the concept of Internet was firstly developed in military bases under its initial name of ARPANET (Established in 1969, the precursor to the Internet was a large wide-area network created by the U.S. Defence Advanced Research Project Agency (ARPA)) [5] and, then, fully developed as an educational concept of interconnection (1) in universities and research centers. The permanent interaction between students and their teachers represents an outstanding priority for the American educational system, which was not the case-culture before 1989. This concept of educational connection implies that each and every computer user can interact with another kind of medium other than the ordinary face-to-face instruction, as for instance, a multimedia technology which is considered an enabling teaching method of presenting computer-based information using varied communicative means, such as text, graphics and sound employed by video and audio technology as it follows: by means of floppies and compact disks, by means of software utilities (in PowerPoint presentations) or by means of the online status. From my point of view, the status of being connected online represents the opportunity of learning about others and getting experience from others at distance.

An important Online European Project, clearly distinguishing itself as a globalization pattern, the well-known Ariadne Project, has been recently presented to University teachers from Romania too, in order to raise pedagogical interest on using new technologies as they allow a well-thought out collaboration among distant users. Consequently, teachers are encouraged to apply a new and more efficient teaching model, which uses tools like discussion forums, e-mailing lists, video-conferencing that represent a pedagogical success in our modern times.

The key words for such an educational enterprise are those that fit to the American way of expressing themselves as a wide insular nation who needs to be open and accessible to the continental diverse cultures. Thus, the Internet is conceived as being open and accessible and, why not, more secure in a way, to every 'internaut' because of the lower costs when accessing the information needed. It is much cheaper and even safer to get connected via cables, phone lines or satellites rather than flying over the ocean. More than that, the linguistic repository on the Internet consists in mere indications of the way the system works. Terms like: 'go to', 'back', 'forward', 'search', 'help', 'home' are at ease and provide self-confidence and re-assurance of not losing yourself in overwhelming data.

\section{Internet, myths, IMs environment and the globalisation concept}

Another aspect of the Internet to be highlighting next is its extensive short form use of net. Therefore, I shall analyze it as related to another kinds of systematic correlations within our experience, a new perspective that brings humanity closer to holiness. To do so, I will be interested to show the remote meanings of the 
word net, starting from the Gospels, in which "nets symbolize divine instrumentality" moving to gather up mankind and bringing "the just into the kingdom of Heaven" [1]). In oriental folklore, the deities make use of holy nets to catch men in their meshes, and in Christian religion, there is a story about two brothers who were called - by God - to be the Fishermen's Souls. In Persian tradition, for instance, the net takes another facet, and this time the opposite of the former analyzed above: it is not the divine tool but of a mundane origin in order to capture a spiritual entity [1].

In our technological society, the appearance of the Internet [5, p.283] is considered a very important event in our lives, having a great impact on human experience, as well as the religious cults have had on humankind along the history. (The Internet has more than 100 million users worldwide, and that number is growing rapidly. More 100 countries are linked into exchanges of data, news and opinions.) Nowadays, the Net has become an important symbol of the globalization concept, since it connects millions of computers and users altogether. The rapid development of the Internet seems promising since a new era of low-costs has taken shape due to the most advanced electronic technology. This change has forwarded an expansion of a global culture in which information is transferred without frontiers in few seconds through mailing lists, forum groups, or chatrooms (Internet Relay Chat).

The concept of globalization via the Internet is perceived as an extended network of human relationships, ideologies and sets of habits and behaviours, all shared in a process of moving toward another kind of future. Moreover, emotions, states, feelings and language have been standardized over the Internet and heavily used under their new linguistic coinages of smileys, emoticons or audibles.

Then, what kind of language is used in the cyberspace? Due to our awareness of the split spaces in cyberspace and, consequently, of the split user groups (chatters, UNIX groups, Mud's groups, etc.), we may be now in the position to differentiate several types of jargons. For instance, computer jargon is different from the Internet jargon, in point of their reference in time, in the sense that computer jargon has earlier appeared and has been constantly developed ever since, and, on the other hand, in point of the users and their affiliation the system. My opinion about this topic is that, since computers and the whole world their development has generated, like the births of the Internet and of the Web, are related to electronically inputs and outputs signals, the jargon used to describe these electronic processes would be thus peculiar to a whole electronic environment comprising the language inputs for processing the data needed. Therefore, we should talk of the jargon of e-language or, better, an e-jargon since it reflects the language used between humans electronically influenced and mediated.

On the other hand, a language of a highly colloquial type consists of either new words or of current ones employed in some new special sense and typically restricted to a particular context or group of people. This is what we generally refer to as slang which was first attested in English in the mid-eighteenth century (The New Oxford Dictionary of English, 2001). 
Because the Internet represents a globally instant online connection between people no matter what their locations are it has developed its own particular language of combining spoken with written forms. This has happened as a consequence of developing such technologies as Multi User Virtual Environments, Internet Relay Chat (developed by Jarkko Oikarinen in Finland in the late 1980s. [5, p.290]), Bulletin Board, Multi User Dungeon worlds, and many others where different social groups - teenagers, gamers, hackers, etc. build up a highly original way of expressing themselves freely without concerning about whatsoever language restriction rules may be. The Internet slang reveals a remarkable expressiveness and creativity in its forms, making considerable use of linguistic innovations and semantic developments such as word importation (guru), derivation, abbreviations (GIGO, asl), clipping, blending (gork, morf, sorg), sound symbolism (gril), metaphors, analogies, accent, etc. My research is based on a fully participating chat activity, initially as a guest and later as a member within this particular culture because it is necessary, due to the nature of chat rooms, to participate as a full member in order to truly understand the normative system of the new society and the defining and sanctioning processes of deviance within the culture.

The reason why the Internet informal language is so expressive, creative and continuously evolving is due to the presence of instant, live and interactive chat systems which are populated by young people whose imagination and most hidden desires (sexual, linguistic, etc.) are so loose in cyberspace since no authority is there to watch for them and impose behavioural or linguistic constraints. Such instances as $P 911$ are funny and, at the same time, linguistically codified because if you do not belong to the chatters' groups you are considered a newbie or a pona (online newcomers) unable to understand their language. (Pona is a chatroom acronym meaning: Person of No Account.) Thus, P911 immediately sets the alarm off and the chatters' subject needs to be dropped at once because the capitalized $p$ stands for 'parents in the room' and 911 is an alluding way to say emergent request for changing the topic. In other words, you either drop the subject, or watch the language. The acronyms POS and POTS suggest the same behavioural awareness because: Parents are looking Over Shoulder.

Other instances of linguistic creativeness are represented by letters and numbers that often become interchangeable. Because of their phonetic appropriateness, 1 is won or e1 is 'everyone'; 2 may be 'too', 'to' or ' $t w o$ ' as in the following combinations: $2 B Z$ (too busy), 2day (today), 2moro, 2G2BT (Too good to be true), $F 2 F$ (face to face), $g 2 g$ (got to go); $3 o a k$ is 'three of a kind'; 4 is 'for', 'four', or a prefix or suffix of 'fore', as in: $b 4$ (before), 4warned (forewarned), $2 B Z 4 U$ (too busy for you); similarly, letters become lexical morphemes by the process of overgeneralization, like $C$ is 'see' and $U$ stands for 'you' in: $I C U$ ('I see you'), $C U S O O N$; $R$ becomes 'are'; the mixed letter and number combination as $L 8 R$ can be read, for instance, 'later'. If you are not an addicted chatter, you can not comprehend any of these lines below:

Chatter one: $g 2 g$ cul gle 1

Chatter two: hagn tc $h b$ 
Here's the translation of what chatters becoming speakers in a real environment are saying:

Speaker one: Got to go. See you later. Good luck, everyone. Speaker two: Have a good night. Take care. Hurry Back.

The extensive use of symbols gets new connotations in chatters' slang. For example, the symbol $\&$ replaces the syntactic coordinator 'and': $b \&$ would be read 'band' or $h \& k$ would be read 'hug and kiss'; the $\%$ symbol stands for double 'o', so if we have an IM (instant message) like $T \% K$ on our screen, we shall read it as 'took'.

To conclude, on the one hand, chatters chiefly create a universal e-language for the sake of shortening their messages in order to maximize and even hide content from other 'peeps'.

On the other hand, it is so obvious that these "netspeaks" [4] have changed the way of talk-transactions within daily chat use because they represent false images of what we know about conversational rules; this means that chatters make use of verbally communicated language features like fluency and impossibility of self-correcting into a medium that requires orthographic knowledge and very fast typing skills. But at the same time, they lack the basics of expressing non-verbally as they would do in real time communication: posture and position, eye contact and gaze direction.

\section{The superpower's fears of losing control or "Pandora's Box" unleashed}

The facts submitted above represent an optimistic point of view over one of the many facets of the Internet, which I need to remind here that it has been first and foremost invented for military use. This cannot but proves that the world's superpower felt insecure and, therefore, was eager to take control over the most powerful tool in the mankind's whole history, Information. However, there is a flipside of downloading and uploading meaningful and, at the same time, useless data onto servers, because there is always someone to be against your actions. Consequently, a new world of users have emerged and attacked the network in different and witty modes of action by hacking the information, cracking codes and data, flaming the newsgroups or contracting the net with viruses. Speaking this time virtually, the Pandora's Box was once again unleashed by the cyberspace 'rebels'.

To this point, another contemporary jargon that is particularly and clearly codified to the non-initiates, but nevertheless important in the e-language, is one that belongs to hackers. They are considered to be a very intelligent, computer enthusiastic and highly restrictive social group acting beyond the limits of AI laws. This has made them a kind of contemporary embodiments of the legendary heroes, like Robin Hood or Baba Novac (a Romanian legendary hero) who seemed to be brought back in the Third Millennium. Since their computer expertise is generally used for illegal purposes (breaking software codes), the language they developed within time have strongly influenced emergent communicative practice; despite the fact that their notorious curiosity to repair or 
find any hard/software problems and codes is not officially recognized as a profession, their love of playing with symbols - the essence of computer programming - makes us speak of their language as being a hackespeak-jargon or hakspek [4] that does not belong to any vocational authorized group but to a highly restrictive computer addicted young persons who pioneered and partially influenced the human communication mediated by computers. Yet, we may unreservedly draw attention to their particularly passionate and extremely resourceful and literate computer abilities.

Consequently, their language is characterised, as it will be further analysed, by a significantly rich vocabulary associated with computers. Similarly to the chatters' innovative way of exchanging communication through various channels, hackers also have their particular and unusual syntactic and word formation rules invented as a result of their secretive computer related actions hidden behind the computer screen.

\subsection{New sense developments}

Over time the use of ware has been further extended so that hackers coined terms such as the following: freeware, happiware, nagware, crippleware, wetware, annoyware, guiltware, shovelware, a.s.o, many of them being anthropomorphisms.

\subsection{Analogy}

Hackers joyfully renamed the following information units: 2 bits (crumb ("a small particle of anything", (Webster's Encyclopedic Unabridged Dictionary. p.349)) and tayste); 3 bits (trit) (one base-three digit (Adobe Reader Format, New Hacker's Dictionary, p.951)), 8 bits (high bit, hobbit (cultural borrowing. (rare) The High Order BIT of a byte) <meta bit), etc. Hobbit, for instance, has been used to represent fantastic beings by one of the most famous writer belonging to the fantasy genre, J.R.R. Tolkien (1892-1973), in his popular sci-fi books, to name here the trilogy Lord of the Rings and The Hobbit.

There is also a hackish trend to refer to snailmail as paper-net simply because hackers consider the postal service slow and unreliable. (It is sometimes spelled as two separate words, snail mail. [5, p.515].) It is no longer part of the hackish vocabulary.) It is interesting to point out the fact that the term under discussion had not existed until the electronic mail became so ubiquitous especially due to its rapid delivery that has totally changed our life. We even witness the way the process of analogy has been simultaneously created and, in its turn, has given birth to a new term just because the source domain did not have its counterpart for differentiating the two ways of sending messages. The analogy thus created asks us to recall the importance of high technology because it serves our purposes faster than other means of communication which have become older and defective in the millennium we live and think. Hackers have thought of every person's ability to use both hands, and that would be ambimouseterous [7, p.22]. Blamestorming, for example, is an evident analogy with the educational brainstorming process which has just failed because: a 
deadline was missed. It also refers to a group discussion to answer the questions why the respective project failed and who is responsible. Hackers are generally known to be rebel young and very intelligent people who like neither linguistic barriers nor cultural impositions. The hackish word domainist [7, p.275] reveals not only puzzle-affixation but it also makes reference to the judgmental communities of racists, sexists, fascists, feminists, etc., in the sense that the word reflects upon some users' judgmental views about the domain of other users' email addresses.

\subsection{Stylistic level}

Hypocorisma - In order to express their irony towards primitive developing software or to simply make fun of other's coinages, hackers retort to childish way of speaking, thus making use of pet and nursery words: bitty box (ibid. p 89) bixie (syn. to emoticon or smiley, in idem. ibid.), nettie (Darrel Ince, 2001), noddie, clippy (the annoying help assistant wizard resembling a paper clip with eyes), comfy (stands for comfortable). More than that, the last one has been transferred and is extensively used in colloquial speech.

\subsection{Lexical choices}

Puzzle-Affixation (the wrong adding of the suffixes) is another way of showing off: mysteriosity, bogosity, ferrosity, obviosity, dubiosity, softy ([IBM] Hardware hackers' term for a software expert who is largely ignorant of the mysteries of hardware. (http://texts02.archive.org/2/texts/jarg422/jarg422.txt)), stiffy (referring to micofloppies whose jackets are too rigid), firmy;

- the noun-forming suffix -itude to erroneously abstract a quality from just about any adjective or noun: winnitude, lossitude, cruftitude, hackitude, lameitude, hackitude, geekitude, wedgitude, wrongitude.

- The extensive use of the Greek noun designator [9] -oid indicates, as David Crystal points out, a poor imitation, a counterfeit, or some otherwise slightly bogus resemblance: nerdoid [7, p.82], computeroid, (a computer sufficiently small, primitive, or incapable as to cause a hacker acute claustrophobia at the thought of developing software on or for it; something similar to a bitty box), keyboid, windoid, modemoid, servoid.

- The letter ' -0 ' has become a suffix with a negative connotation in hackerese. When added to either verbs or nouns, the respective lexical item points to an error as in: mouso (An error in mouse usage resulting in an inappropriate selection or graphic garbage on the screen. (The Jargon Files, http://www.houghi.org/jargon/mouso.php)), braino, scanno, thinko (a momentary, correctable glitch in mental processing, especially one involving recall of information. (http://texts02.archive.org/2/texts/jarg422/jarg422.txt)), typo, blammo (to forcibly remove someone who is misbehaving from any interactive system, especially talker systems), blendo page. (It is normally used to describe a poorly designed web page. (Darrel Ince, op.cit., p.33.)) 
However, things happen differently at the semantic level when talking of the slangy 'compo', which means competitions for all kinds of demos, like: music compos, graphic compos.

Less common but still 'alive' is the verbal form disuser (wrong affixation and weird conversion), meaning the removal of a user's account.

Conversion - Also, hackers cheerfully consider that nouns can be converted into verbs, such as to geek out (to talk technically, [5, p.84]) $(<\mathrm{n}$. geek), or other instances as:

1. I'll mouse it up.

2. Hang on while I clipboard it over.

3. I'm grepping the files.

4. Why don't you webify all your publications? ( $<$ the noun web).

5. A program that has been changed so many times as to become completely disorganized has become bogotified. ( $<$ the noun bogus).

6 . His talk was relatively sane until somebody asked him a trick question; then he bogued out and did nothing but flame afterwards. (The Jargon Files, http://www.houghi.org/jargon/bogue-out.phpda.)

7. He got disusered when they found out he'd been cracking through the school's Internet access. [7, p.271].

8. It just is obvious that hackers are ahead of this general trend of categorial change as they use this technique very generously. Many verbs are constructed by taking the name of a computer utility: to tex, to lint, to wall, etc.

9. Some verbs change their roots as in: hackify $>$ hackification [4].

10. There are instances when computer nouns are converted into adverbs, like the well-known software/hardware that becomes softwarily/hardwarily (a software-related problem. ((http://texts02.archive.org/2/texts/jarg422/ jarg422.txt)) when there is a problem with the software/hardware.

Blending has started as one of the most productive lexical processes in commercial coinages and continued to develop and extremely grow in popularity within the computer world; therefore, hackers seem to take pleasure in the use of blends, too. The following words may be considered: hackitude, nugry, (http://developer.syndetic.org/query_jargon.pl?term=newbie), Sysprog, gensym, etc.

\subsection{Cultural borrowings}

Hackers borrow cultural beliefs and behaviour that suit to their activities. Words like zen, kamikaze, samurai come from the Japanese culture and art of war; kahuna, or guru, which is known to be a Hindu spiritual mentor or guide [6], gets new connotations when referring to a person with particular expertise or knowledge; both terms are imported from Hawaiian and Indian myths and beliefs. Samizdat points to the Russian communism and hobbit is of AngloSaxon origin. 


\section{Conclusions}

To conclude, many hackish expressions are as much representation of a special way of thinking of the world, maybe even a distinctive way of consciousness. This consciousness has its roots mainly in the Hackish Culture, which seems to be a mixture of Mathematics with Physics, like computron, bogon invented by analogy with proton, electron, neutron, etc. (Carroll, M.C. Escher, Magritte) and not very irrational but very improbable elements, like obi-wan error (hint at the "Star Wars" science-fiction movie).

Secondly, the language they use does not reflect lack of some grammar knowledge; they only want to create new words on the spur of the moment and deliberately and consciously use non-standard English morphology - often without even the need for the new word as a motivation but for the process itself. In doing this, a certain degree of originality seems to be the criteria to build these new words considered as linguistic innovations and forms of playfulness purposefully created to amuse and to come up with novel ways, never at the expense of clarity, to characterize each others' habits and style of interacting with programming code and technology. Therefore, their study is useful for getting to know other fresh aspects of language.

On a psychological level, it is worth mentioning that many studies of on-line communication have shown that electronic links have a de-inhibiting effect on people. Deprived of the body-language cues through which emotional state is expressed, people tend to forget everything about other parties except what is presented over that ASCII link, and this has especially positive effects. For instance, it encourages honesty and tends to break down hierarchical authority relationships.

Finally, the use of excessively specialized, encoded vocabulary helps them hold a hackish culture in a restricted space as they recognize each other's places in the community by sharing mutual values and experiences. Using their terminology in an inappropriate manner, defines you as an intruder in their own world.

An overall conclusion of this paper must be drawn and we must say that the above discussions point to further specialization in computer-mediated discourse whether it reflects "netspeaks", "teckspeaks" or "hackespeaks", and to potentially build on more comprehensive theories of social and cultural interaction among them. More than that, these "netspeaks" are informal as we have already demonstrated.

More than that, the paper forwards a special (novel and above regulated common norms) way of thinking of the world in its fully active interaction with a new technology and its major participants: the Third Millenium teenagers and young people. Therefore, the e-language analysed may be characterised, above all, as extremely original and spontaneous due to the fact that what is considered non-standard English in day by day life has become standardized in virtual world.

By creating the Web, a new cultural bridge has been built over the Atlantic Ocean and the whole world (especially Europe, starting from T. B. Lee, the Web 
inventor). Moreover, cultures all over the world experience the ongoing process of being re-engineered and reconfigured under the impact of this new technology.

Last but not least, the various online communities have learned to adapt new ways of command, to acknowledge power relations, and to have respect for skills and status (available, busy, on the phone, idle, invisible, etc.). As seen, deceit, duplicity, and disguise (nicks, charcters, hosts) have all become possible in the chat-texting world as the 'keyboard generations' (credits to Dr. Randall, Lingo Online: The Language of the Keyboard Generation. 2002) and their environments have developed various strategies in representing and interpreting their actions because they are naturally "adapting to online communication and as a result have learned to speak with their fingers" (Dr. Randall, 2002).

\section{References}

[1] Chevallier, Jean and Gheerbrant, Alain. A Dictionary of Symbols, BLACKWELL Reference, USA: 1994.

[2] Herring, Susan. Computer-Mediated Communication. Linguistic, Social Cross-Cultural Perspectives. John Benjamins Publishing Company, Amsterdam/Philadelphia: 1996.

[3] Crystal, David. Language and the Internet, Cambridge University Press: 2001.

[4] Crystal, David. A Glossary of Netspeak and of Textspeak. Edinburgh University Press: 2005.

[5] Margolis, Philip E.. Computer \& Internet Dictionary, Random House Webster, $3^{\text {rd }}$ Edition: 1999.

[6] Cassell's Foreign Words and Phrases, London, Cassell's Co Wellington House: 2000.

[7] Raymond, Eric S. http://www.logophilia.com/jargon/jargon_5.html .1998

[8] Teleoacă, Anca Irinel. 2004. Culturally-Embeded Concept via Internet. The Unifying Aspects of Cultures. TRANS. No. 15. URL: http://www.inst.at/trans/15Nr/08 4/teleoaca15.htm

[9] Fuzzy Grammar, OUP. 2004 